\title{
Analysis and Improvement of Routing Protocol LEACH using TEEN, APTEEN and Adaptive Threshold in WSN
}

\author{
Bhakti Parmar \\ Department of Electronics and Communication \\ Engineering, UKA TARSADIA University, Gujarat, \\ India.
}

\begin{abstract}
A wireless sensor network consists of spatially distributed sensor nodes which sense physical and environmental condition like sound, pressure, temperature etc and pass the data to the main location through the network. The recent advances and improvement of micro electro-mechanical systems technology, integrated circuit technologies, microprocessor hardware, wireless communications, Ad-hoc networking routing protocols and embedded systems have made the concept of Wireless Sensor Networks. In WSN network life time and node energy efficiency are two most important terms. The aim of this study is to making an energyefficient routing protocol which has a significant improvement on the overall lifetime of the sensor network and energy efficiency of nodes. LEACH is energy-efficient hierarchical based protocol that balances the energy efficiency, saves the node energy and hence the lifetime of the network. But Because of certain limitations of routing protocol LEACH, some schemes are proposed using protocols TEEN and APTEEN to overcome the drawback of LEACH. But TEEN and APTEEN has also some drawback and that drawback is removed by an advance scheme that is ADAPTIVE THRESHOLD. It gives better energy efficiency and improved network life time compared to LEACH, TEEN and APTEEN.
\end{abstract}

\section{Key Words}

Wireless Sensor Networks, Routing protocol LEACH, TEEN, APTEEN, ADAPTIVE THRESHOLD, Node scheduling, Energy efficiency, Network lifetime

\section{INTRODUCTION}

Wireless Sensor network is the most promising tool for checking the physical conditions such as sound, pressure, temperature, humidity, intensity, vibration, motion, pollutants etc. at different locations. It also uses self-organizing networks which have the capacity of sensing, processing and communicating. A sensor network [1] [4] is a network of so many small disposable low power devices, called nodes, which are spatially distributed in order to perform an application-oriented global task. Different types of routing protocols with its features are discussed here.

LEACH is an energy awaked cluster based routing protocol which reduces the energy of WSN by randomizing cluster heads during life span of network. However, normal LEACH protocol is still having lots of limitations. Many techniques proposed as new modification for LEACH to provide more lifetime and to reduce energy consumption. LEACH modifications include improved clustering methods, advanced routing techniques, energy level assignment, data aggregation and decrement of redundant nodes with proper node scheduling techniques of active and sleep modes in each

\author{
Jayesh Munjani \\ Assistant Professor, Department of Electronics \\ and Communication Engineering, UKA TARSADIA \\ University, Gujarat, India
}

cluster at a periodic time interval in WSN. The modes are decided according to the condition given by protocols TEEN, APTEEN and ADAPTIVE THRESHOLD.

\section{RADIO MODEL OF LEACH}

The first order radio model is used in LEACH. The energy cost for transmit and receive k-bit message between two nodes separated by distance of $\mathrm{d}$ meters in LEACH given by equation

$$
\begin{aligned}
\mathrm{E}_{\mathrm{TX}}(\mathrm{k}, \mathrm{d}) & =\mathrm{kE}_{\text {elec }}+\mathrm{k} \varepsilon_{\mathrm{fs}} \mathrm{d}^{2}, \text { if } \mathrm{d}<\mathrm{d}_{0} \ldots \ldots \ldots .(1) \\
& =\mathrm{kE}_{\text {elec }}+\mathrm{k} \varepsilon_{\mathrm{mp}} \mathrm{d}^{2}, \text { ifd }>=\mathrm{d}_{0} \ldots \ldots \ldots . .(2) \\
\operatorname{ERx}(\mathrm{d}) & =\mathrm{kE}_{\text {elec }} \ldots \ldots \ldots \ldots \ldots \ldots \ldots \ldots \ldots(3)
\end{aligned}
$$

Where $E_{T X}$ in $E q(1)$ denotes the total energy used in the transmitter of the source node, and $\mathrm{E}_{\mathrm{RX}}$ in $\mathrm{Eq}$ (3) represents the energy used in the receiver of the destination node. $\varepsilon_{\mathrm{mp}}$ is the energy needed by the transmit amplifier to manage suitable signal-to-noise ratio in order to transfer data messages properly.

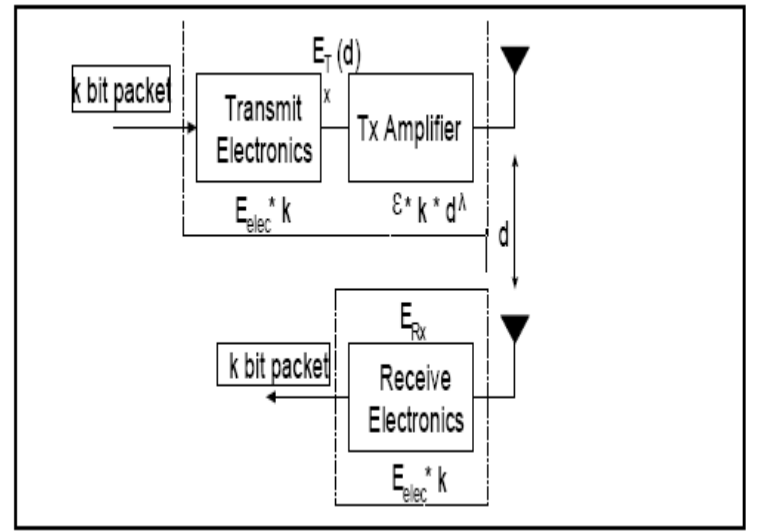

Figure 1:First Order Radio Energy Model[5]

As is the case in, LEACH uses both model the free-space propagation model and the two-ray ground propagation model to count the path loss sustained due to wireless channel transmission. Depending on the distance between the transmitter and receiver, both the free space (d2 power loss) and the multi path fading ( $\mathrm{d} 4$ power loss) channel models are used. If the distance is more than a threshold, the multi path model is used; otherwise, the free space model is used. $d_{0}$ is the distance threshold given by

$\mathrm{d}_{0}=\operatorname{sqrt}\left(\varepsilon_{\mathrm{fs}} / \varepsilon_{\mathrm{mp}}\right)$ 


\section{LEACH- NODE SCHEDULING 3.1.LEACH}

Normal LEACH is proactive network protocol. It checks the network and gives the results periodically. It is most appropriate only for constant monitoring of networks. In most cases, the user does not always need all that data, therefore periodic data transmission are useless. It consumes more energy. To reduce the drawback of normal LEACH, LEACH node scheduling using TEEN and APTEEN used.

Node scheduling is clustering based data gathering approach which is having the node scheduling of active and sleep modes in each cluster at a periodic time interval or at each cluster change time in the wireless sensor networks to increase the lifetime of sensor nodes and energy of network. Here the formation of cluster and cluster head is same as in normal LEACH protocol. After the formation of cluster head when there is a procedure of scheduling nodes it differs from normal leach. Clusters are formed with the nodes which have two modes one is active mode and another is sleep mode. The modes are decided according to the condition given by two protocols TEEN \& APTEEN. In each cluster, condition will checked and if the condition will satisfied than according to the given condition, the nodes are keeping in active mode and in sleep mode for certain time intervals. Whenever the nodes are in sleep mode in each cluster it consumes no energy. After that certain time the condition will again checked and again the modes are decided. The condition is based on Hard threshold (HT) and Soft threshold (ST).

Hard threshold (HT): Hard threshold is a threshold value for the first sensed attributes developed for reactive networks. It is the threshold value of the sensed attributes beyond which the node sensing this value must switch on its transmitter and transmit to its $\mathrm{CH}$.

Soft threshold (ST): Soft threshold is little change in the value of the sensed attributes that triggers the node to switch on its transmitter and than transmit to it's $\mathrm{CH}$.

\subsection{LEACH- Node Scheduling using TEEN}

A reactive network protocol called TEEN is Thresholdsensitive Energy Efficient sensor Network. In Reactive Networks, sensor nodes sense the environment periodically and transmit the value when the value exceeds a user specified threshold value [6] that means when sensed parameter is critical. In this scheme, at each and every periodic time interval, in addition to the attributes, the $\mathrm{CH}$ broadcast $\mathrm{HT}$ and ST to its members. The nodes sense environment at regular basis. When the sensed parameter from the attribute set reaches its hard threshold value, the node switches on its transmitter and transmits the sensed information. An internal variable in the node stores the sensed value called SV. The nodes will next transmit the information in the current cluster period only when both the following conditions are correct.

1. The current sensed value of the sensed attribute is greater than previous sensed value that is HT.2. The current sensed value of the sensed attribute differs from previously stored sensed value (SV) by an amount equal to greater than the small change (ST).

The HT tries to reduce the number of transmission by allowing the nodes to transmit only when the sensed attribute is in the range of interest. The ST further reduces the number of transmissions by eliminating all the transmissions which have occurred because of little or no change in the sensed attribute once the HT decided. Energy consumption in this scheme can be much less than in proactive network that is normal LEACH. Because data transmission consumes more energy than data sensing and in this scheme data transmission is done less frequently. Advantage of this scheme is it is best suited for time critical data sensing application.

But the main drawback of this algorithm is that the nodes will not communicate with each other, if the thresholds are not reached and the user will not get any information about the network situation, and can not know even if the nodes will die. Therefore this scheme is not suited for applications where it is necessary to get data on a regular basis. To overcome the drawback of TEEN, advance protocol APTEEN is used.

\subsection{LEACH-Node Scheduling using APTEEN}

A hybrid network protocol called APTEEN known as a Adaptive periodic threshold sensitive energy efficient sensor network protocol. Hybrid Networks gives the combination of best features of proactive and reactive networks, while reducing their drawbacks.

The user might need a network that reacts immediately to major changes in data and gives information about all situation of network periodically, so that it is able to answer analysis queries. None of the above sensor networks can do both jobs properly since they have their own limitations. APTEEN is combination of the proactive and reactive networks while reducing their drawbacks to create a new network called a hybrid network. In this network, the nodes not only send data periodically, they also respond to sudden changes in attribute values. In this way it works as a proactive protocol as well as reactive protocol. Data values exceeding the threshold value are known as critical data. The nodes monitors their environment at each and every time. Only those nodes which sense the value at or beyond the hard threshold can transmit. Furthermore, the next transmission will done after once a node senses a value beyond HT only when the value of that attributes changes by an amount equal to or greater than the ST.

The exception to this rule is that a node is forced to sense and transmit the data to get the information about the network, irrespective to the sensed value of the attribute, if it does not send data for time period equal to the count time. But it consumes more energy, so another scheme ADAPTIVE THRESHOLD proposed.

\section{PROPOSED PROTOCOL ADAPTIVE THRESHOLD}

ADAPTIVE THRESHOLD is a variable threshold in which value of threshold get changed after predefined round. ADAPTIVE THRESHOLD is variance of past sensed values. Constant soft threshold is the draw back of protocol TEEN and APTEEN because it gives the limited results. In ADAPTIVE THRESHOLD the soft threshold is not constant but varying with the sensed values. The sensed values are as per the count time, and the variations are taken by averaging (as per eq.4) these sensed values and then taking variance (as per eq.5)of these sensed values. These variations work as a soft threshold.

Avg. $=$ sum of all sensed values/total sensed values ...(4)

Variance $=$ Square root of (sum of (sensed value avg.) $)^{2)} /$ count ...........(5) 
Because of the variations in soft threshold, user gets a complete picture of the network at least one time in a count time. The node will be active at least one time as the soft threshold is the variance of all those sensed values and the node become active if it is in the range of the soft threshold. In this way the drawback of TEEN and APTEEN will overcome. The similar data that means the sensed value with little or no changes will not be transmitted to the $\mathrm{CH}$ so node is in sleep, there is no transmission. So the life time of nodes will increase and get better energy efficiency.

\section{SIMULATION AND RESULTS}

The network includes some of the initial simulation parameters and the initialization of the sensor network. So it is necessary to generate the locations of the nodes in the $\mathrm{L} * \mathrm{~L}$ $\mathrm{m} 2$ of the region. Random 100- node topology for a $100 * 100$ $\mathrm{m} 2$. region, base station is located at $(50,50)$. The simulation parameters used in the experiment is shown below:

Number of sensor nodes (N) - 100

Network area (MxM) - 100 x 100 m

Location of base station $(\mathrm{x}, \mathrm{y})-(50,50)$

Eelec (transmission \& reception energy per bit) - 50 $\mathrm{nJ} / \mathrm{bit}$

Eamp (amplification energy at transmitter per bit) $0.0013 \mathrm{pJ} / \mathrm{bit} / \mathrm{m}^{4}$

Eda (data aggregation energy per bit) - 0.5J

$\mathrm{K}$ (number of bits in a packet) - 4000bits

Constant for free space energy $-10 \mathrm{pJ} / \mathrm{bits}^{2}$

For comparisons of normal LEACH with the improved LEACH the some results are taken. In case of proposed protocol giving the simulation result of alive nodes after 1000 rounds are more than the normal LEACH. After 1000 rounds normal LEACH has 39 dead nodes and remaining energy is near by $55 \mathrm{~J}$ out of $200 \mathrm{~J}$, APTEEN has 41 dead nodes but remaining energy is about $77 \mathrm{~J}$ which is more than $\mathrm{LEACH}$. TEEN has 26 dead nodes remaining energy is about 109J which is more than both LEACH and APTEEN and ADAPTIVE THRESHOLD has 20 dead nodes and $119 \mathrm{~J}$ remaining energy which is the best result compare to LEACH, TEEN and APTEEN.

Table - I: Performance comparison of protocols for 1000 rounds

\begin{tabular}{|c|c|c|c|}
\hline Protocols & $\begin{array}{c}\text { No. of } \\
\text { rounds } \\
\text { used }\end{array}$ & $\begin{array}{c}\text { Total } \\
\text { dead } \\
\text { nodes } \\
\text { (out of } \\
\text { 100 node) }\end{array}$ & $\begin{array}{c}\text { Total } \\
\text { remaining } \\
\text { energy } \\
\text { (out of } \\
\mathbf{2 0 0 J})\end{array}$ \\
\hline LEACH & 1000 & 39 & 55.1915 \\
\hline TEEN & 1000 & 26 & 109.4239 \\
\hline APTEEN & 1000 & 41 & 77.8474 \\
\hline ADPTV.TH. & 1000 & 20 & 119.1328 \\
\hline
\end{tabular}

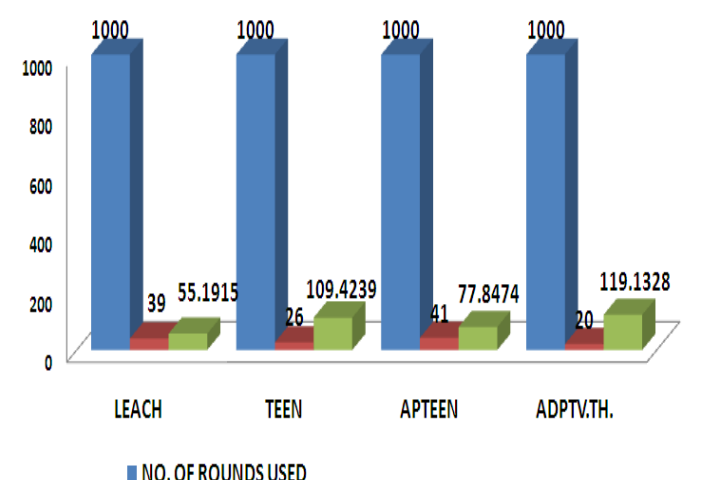

I TOTAL DEAD NODES(Lifetime of network) (out of 100nodes)

- TOTAL REMAINING ENERGY(OUTOF 200J)

Figure 2: Comparison for 1000 rounds

After 2000 rounds all the nodes become dead in normal $\mathrm{LEACH}$, it runs up to 1853 rounds only and after completion of 1853 rounds remaining energy is also almost zero. APTEEN has 50 dead nodes and $34 \mathrm{~J}$ remaining energy. TEEN has 45 dead nodes and $54 \mathrm{~J}$ remaining energy. ADAPTIVE THRESHOLD has 38 dead nodes and $73 \mathrm{~J}$ remaining energy after 2000 rounds which is the best result again.

Table - II: Performance comparison of protocols for 2000 rounds

\begin{tabular}{|c|c|c|c|}
\hline Protocols & $\begin{array}{c}\text { No. of } \\
\text { rounds } \\
\text { used }\end{array}$ & $\begin{array}{c}\text { Total } \\
\text { dead } \\
\text { nodes } \\
\text { (out of } \\
\mathbf{1 0 0} \text { node) }\end{array}$ & $\begin{array}{c}\text { Total } \\
\text { remaining } \\
\text { energy } \\
\text { (out of } \\
\text { 200J) }\end{array}$ \\
\hline LEACH & 1853 & 97 & 0.0223 \\
\hline TEEN & 2000 & 45 & 54.6208 \\
\hline APTEEN & 2000 & 50 & 34.1816 \\
\hline ADPTV.TH. & 2000 & 38 & 73.3942 \\
\hline
\end{tabular}

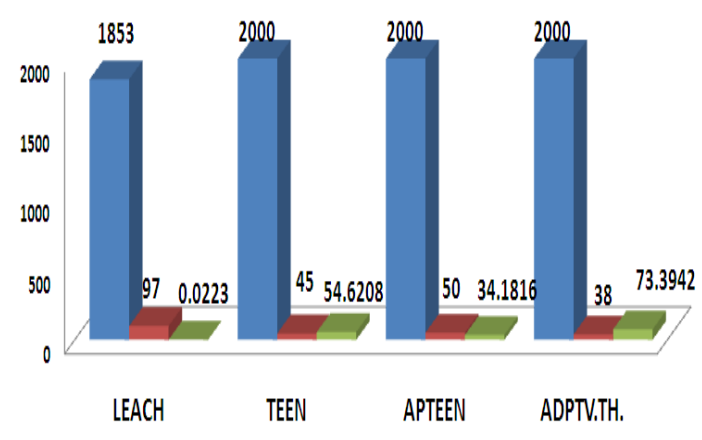

INO, OF ROUNDS USED

I TOTAL DEAD NODES(Lifetime of network)(out of 100 nodes)

I TOTAL REMAINING ENERGY(OUTOF200J)

Figure 3:. Comparison for 2000 rounds 
Each protocol runs up to different rounds so the maximum rounds covered by each protocol are as follow:

\section{LEACH - 1853 \\ APTEEN - 3803}

\section{TEEN - 4311}

\section{AD.TH.- 6518}

All the nodes become dead and energy get zero after completion of 1853 rounds in LEACH, 3803 rounds in APTEEN, 4311 rounds in TEEN and 6518 rounds in ADAPTIVE THRESHOLD. So ADAPTIVE THRESHOLD gives the best results compare to LEACH, TEEN and APTEEN.

Table - III: Performance comparison of protocols for maximum rounds

\begin{tabular}{|c|c|c|c|}
\hline Protocols & $\begin{array}{c}\text { No. of } \\
\text { rounds } \\
\text { used }\end{array}$ & $\begin{array}{c}\text { Total } \\
\text { dead } \\
\text { nodes } \\
\text { (out of } \\
\mathbf{1 0 0} \text { node) }\end{array}$ & $\begin{array}{c}\text { Total } \\
\text { remaining } \\
\text { energy } \\
\text { (out of } \\
\text { 200J) }\end{array}$ \\
\hline LEACH & 1853 & 97 & 0.0223 \\
\hline TEEN & 4311 & 99 & 0.0076 \\
\hline APTEEN & 3803 & 99 & 0.0050 \\
\hline ADPTV.TH. & 6518 & 99 & 0.0050 \\
\hline
\end{tabular}

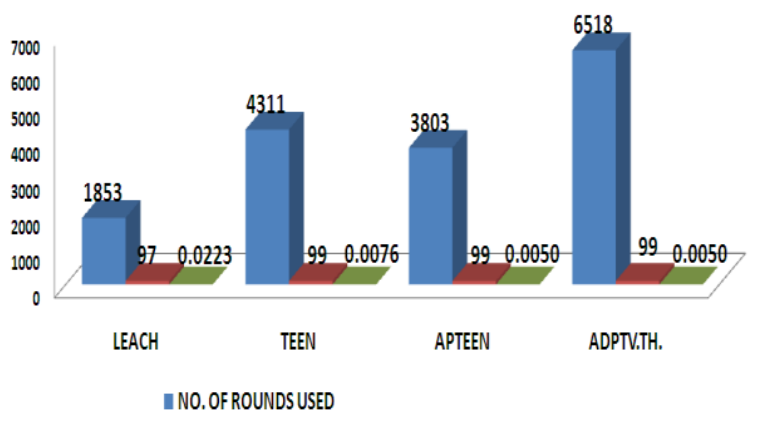

I TOTAL DEAD NODES(Lifetime of network) (out of 100 nodes)

I TOTAL REMAINING ENERGY(OUTOF 200J)

Figure 4:. Comparison for maximum rounds

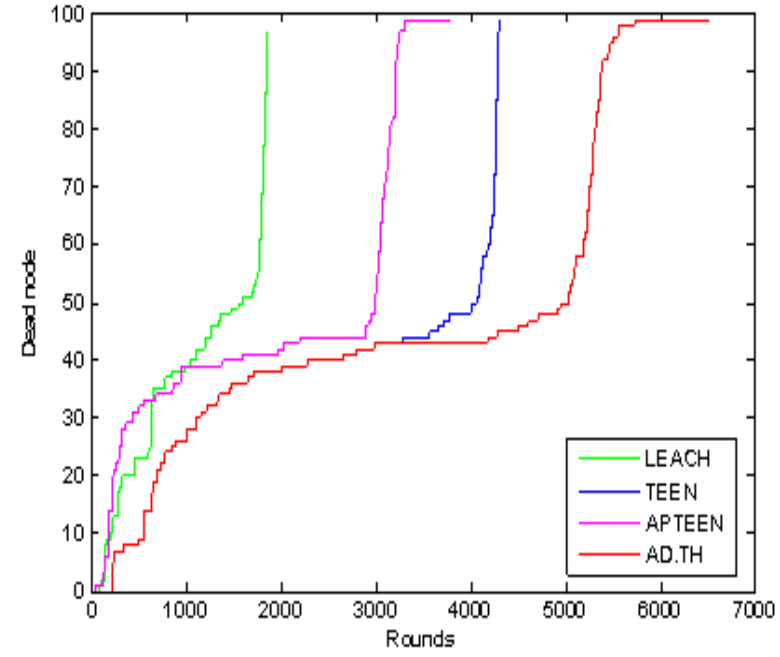

Figure 5: Round Vs. Dead nodes for max. rounds

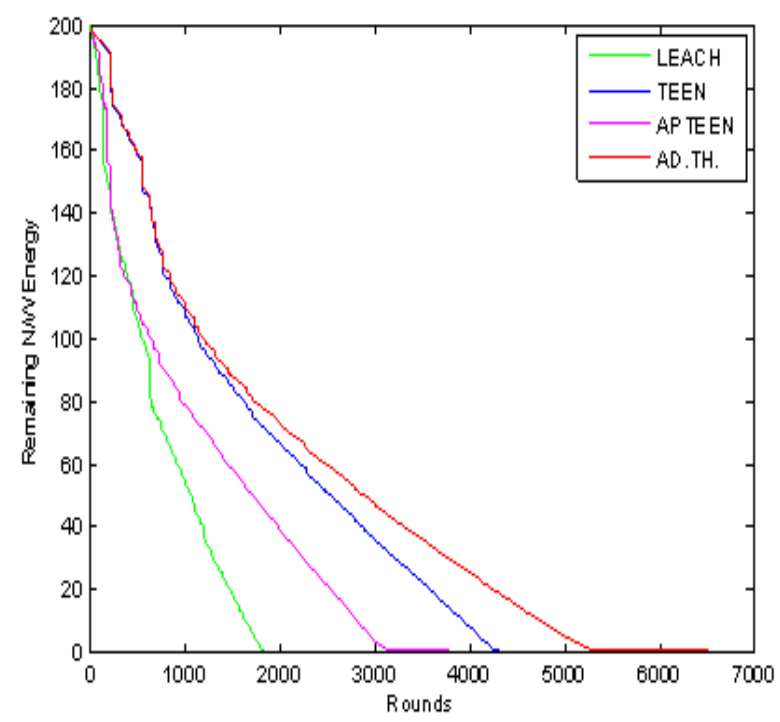

Figure 6: Round Vs Remaining N/W Energy for max. rounds

\section{Conclusion}

The proposed protocol is presented using the proper node scheduling (ACTIVE and SLEEP) in the individual clustering of the whole network. Clustering is according to the condition given by TEEN, APTEEN and ADAPTIVE THRESHOLD based on ST and HT. Because of less transmission energy consumption will decrease and network lifetime will increased. APTEEN works better than LEACH.TEEN gives better results than APTEEN and LEACH both. And the most improved results are achieved by ADAPTIVE THRESHOLD. IN ADAPTIVE THRESHOLD algorithm, threshold is changed based on previously sensed data. So sensor will try to adapt the environment changes by varying the threshold. So the conclusion is that ADAPTIVE THRESHOLD gives better results, improved energy efficiency of nodes and better network lifetime compared to normal LEACH, TEEN and APTEEN.

\section{REFRENCES}

[1].Ian F. Akyildiz, Weilian Su, YogeshSankaraubramaniam, and ErdalCayirci: A Survey on sensor networks, IEEE Communications Magazine (2002). 
[2].José A. Gutierrez, Marco Naeve, Ed Callaway,MoniqueBourgeois,VinayMitter, Bob Heile, IEEE 802.15.4: A Developing Standard for Low-Power Low-Cost Wireless Personal Area Networks, IEEENetwork, pp. 12-19 (September/October 2001).

[3] .Ed Callaway, Paul Gorday, Lance Hester, Jose A. Gutierrez, Marco Naeve, Bob Heile, VenkatBahl: A Developing Standard for Low-Rate Wireless Personal Area Networks; IEEE Communications Magazine, pp. 70-77 (August 2002).

[4].Sarjoun S. Doumit, Dharma P. Agrawal: Self- Organizing and Energy-Efficient Network of Sensors, IEEE, pp. 1-6 (2002).

[5].W.R.Heinzelman, Energy-efficient Communication protocol for Wireless microsensor networks, in: Proc. of 33rd Annual Hawaii Inter Cord on System Sciences, Hawaii, and USA: IEEE Computer Society, 2000.

[6].By Dharam Prakash Agarwal Qing, An Zeng Cengage "Introduction to Wireless and Mobile system", Second Edition, Ch-13, Page No 303 - 354,

\section{ACKNOWLEDGEMENT}

I would like to thank my guide Prof. Jayesh Munjani for their guidance and encouragement in the development and preparation of this dissertation. Without them this thesis would not exist.

BIOGRAPHIES AUTHOR: JAYESH MUNJANI, Assistant Professor, Department of Electronics and Communication Engineering, UKA TARSADIA University, MALI BA campus, bardoli, 